\title{
ANTAGONISMO ENTRE PRINCIPIOS INTERNACIONALES EN LA CRISIS DE VENEZUELA
}

\author{
Tony Altamirano Andino ${ }^{1}$
}

\section{RESUMEN:}

El presente artículo científico pretende desarrollarse desde un enfoque analítico reflexivo para brindar un panorama amplío y significativo en cuanto a la situación jurídica en la rama del derecho internacional que atraviesa la República Bolivariana de Venezuela, en cuanto a la repercusión que puede propiciar las distintas políticas exteriores e intereses particulares de los actores internacionales como lo es China, Rusia y Estados Unidos en la crisis interna de esta nación sudamericana, partiendo el análisis de determinar los principios internacionales que estas potencias mundiales determinan en aplicar como parte de sus políticas exteriores hacia el concierto mundial de naciones, en relación a la crisis política interna de Venezuela, ante el supuesto desabastecimiento de los insumos mínimos al que tiene derecho toda persona en el ámbito de los derechos humanos.

Se desarrollará en el mismo orden de idea de la temática, el fenómeno evolutivo del principio de unidad territorial de un Estado como lo determina la Resolución 1514 promulgado por la Asamblea General de las Naciones Unidas, en cuanto a la relación intrínseca de unidad nacional, estrictamente en la unidad política de un país, refiriéndonos al innovador fenómeno denominado hipótesis internacional de dualidad política en el marco legal de la autodeterminación externa de un país hacia otro, teniendo como punto de enfoque para el desarrollo de estos puntos, la situación jurídico-político en el ámbito del derecho internacional en el caso de la crisis o conflicto interno de la República Bolivariana de Venezuela que genera el antagonismo entre el principio de autodeterminación de los pueblos y el principio de responsabilidad de proteger.

PAlabras ClaVE: Naciones Unidas (ONU), Resolución 1514, Principio de Responsabilidad de Proteger, Principio de Autodeterminación de los Pueblos, Carta de las Naciones Unidas, Tribunal Supremo de Canadá, Misión Observación Electoral de la Organización de Estados Americanos (OEA). Crisis de Venezuela.

Fecha de recepción: 31 de agosto del 2019

Fecha de aprobación: 15 de octubre del 2019

\footnotetext{
${ }^{1}$ Master en Derecho Internacional y Relaciones Internacionales de la Universidad Complutense de Madrid, España. Abogado egresado de la Universidad Nacional Autónoma de Honduras. Asesor y Director en Juicio del Consultorio Jurídico Gratuito, unidad académica adscrita a la Faculta de Ciencias Jurídicas de la Universidad Nacional Autónoma de Honduras. Correo electrónico: tony.altamirano@unah.edu.hn
} 


\title{
ANTAGONISM BETWEEN INTERNATIONAL LAW PRINCIPLES IN THE VENEZUELAN CRISIS
}

\begin{abstract}
The scientific article pretends to develop from an analytic-reflexive point of view, to provide a broad and significative panorama attending the actual juridical situation in the branch of the international law that affects the Bolivarian Republic of Venezuela, considering the repercussion that can arouse the foreign policies and particular interests that the international actors like China, Russia and the United States of America have in the internal crisis of this south american nation, delimiting our analysis to determine the international law principles that these global powered nations determine to apply in there foreign policies towards the international community of nations based on the internal political entanglement of Venezuela, taking in consideration the supposed shortage of minimal inputs that every human being is entitled to in the field of human rights.
\end{abstract}

In the same order of ideas of our thematic, will be developed taking in consideration the evolutive phenomenon of the territorial unity of a State as it is determined by the 1514 Resolution promulgated by the General Assembly of the United Nations, focusing in the intrinsic relation of the national unity within the political unity of a country, referring to the innovative international phenomenon denominated hypothesis of political duality in the legal framework of the external auto determination from one country to another, focusing the analysis the political-legal point of the view of the international law in the internal crisis or conflict of the Bolivarian Republic of Venezuela that generates the antagonism between the principle of self-determination of the people and the principle of responsibility to protect.

KEYWORDS: United Nations (UN), 1514 Resolution, Responsibility to Protect Principle, Self Determination of the People Principle, United Nations Letter, Supreme Tribunal of Canada, Electoral Observatory Mission, Organization of American States (OAS), crisis of Venezuela.

Date of receipt: August 31, 2019

Approval date: October 15, 2019

\footnotetext{
${ }^{2}$ Master Degree in International Law and International Relations of the Complutense University of Madrid, Spain. Lawyer degree from the National Autonomous University of Honduras. Adviser and Director in trials of the Free Legal Bureau, academic unit associated to the Law School Faculty of the National Autonomous University of Honduras. Email: tony.altamirano@unah.edu.hn
} 


\section{INTRODUCCIÓN}

El génesis del derecho internacional propiamente como tal, es a partir de la creación de la Carta de las Naciones Unidas con su firma el 26 de junio de 1945, como consecuencia directa de los hechos posteriores al término de la II Guerra Mundial, creando el máximo órgano internacional regulador del derecho internacional.

En este cuerpo jurídico encontramos múltiples principios que rigen la relación entre los Estados partes del concierto internacional, que a través de estos 74 años de vida, estos han tenido múltiples cambios y mutaciones dependiendo la situación jurídica individual de cada Estado que ha gozado o ha sido afecto a acciones tanto beligerantes con el uso de la fuerza armada por las potencias mundiales, así como políticas internacionales coercitivas sin el uso de la fuerza armada como sanciones económicas o embargos comerciales para que un Estado cese en sus actividades consideradas internacionalmente ilícitas.

Si bien es cierto que el derecho internacional ha tenido una evolución significativa en el último siglo, comprendemos que los principios reguladores de las relaciones internacionales continúan teniendo una preponderancia determinante para regular o determinar un hecho internacionalmente ilícito en relación a delitos de lesa humanidad o que atenten contra la integridad física y moral de los ciudadanos de un Estado en la esfera de los derechos humanos.

En la crisis interna venezolana, emergen muchos factores que incitan a que potencias como Estados Unidos, China y Rusia intervengan con tesis antagónicas del derecho internacional para poder tener un provecho ya sea comercial, político para concesión de explotación de recursos naturales, intereses estratégico militares, dando lugar en este caso en concreto, la implementación de la secesión política de un Estado, fenómeno internacional novedoso (del Arenal Moyua, 2007, pág. 350), nunca antes visto en el tiempo de vida del derecho internacional, pudiéndose ver como un antónimo de la unidad nacional, trasladándose al ámbito político con la dualidad de la figura presidencial en Venezuela, reconocido por unos países, y no reconocido por otros.

En cuanto a los principios de libre determinación de los pueblos y el principio de responsabilidad de proteger, será el pilar central del análisis reflexivo a desarrollar, para poder enunciar el antagonismo directo entre estos 2 principios en la crisis jurídica a lo interno, y en el ámbito internacional en Venezuela, así como la preponderancia que posee cada principio, ya que no existe un orden de preminencia jurídica internacional detallado taxativamente en un tratado internacional o en algún cuerpo jurídico de orden internacional, siendo en este caso, la costumbre internacional la que determina la preponderancia de uno de estos principios, teniendo como punto central, el poder esclarecer de acuerdo a una de las fuentes del derecho internacional como lo es la costumbre, el principio que prima en detrimento del otro.

\section{METODOLOGÍA}

La investigación a desarrollar, reúne las características de analítica, descriptiva y explicativa, desde una perspectiva u orden teórico, no abarcando el factor de investigación experimental. Se utilizó el método de investigación descriptivo, por cuanto se fundamentó en dos aspectos jurídicos internacionales que analizan diversos elementos evolutivos objeto de estudio en relación a los próximos conflictos internacionales que puedan suscitarse. Se utilizó también la metodología analítica y explicativa por desarrollarse los efectos colaterales y preponderancia que los principios internacionales de libre determinación de los pueblos y la responsabilidad de proteger tienen ahora mismo en Venezuela, exponiendo de manera exhaustiva las problemáticas, causas, fenómenos e impacto directo de la crisis interna de Venezuela con efectos y repercusiones en la jurisprudencia internacional. 


\section{Principio de Libre Autodeterminación de los Pueblos}

La definición de este principio universal del derecho internacional nació con la intención de proteger a pueblos o territorios sin autogobierno que históricamente han sido sometidos a esclavitud o procesos de colonización. Al hablar de este término, su génesis se establece en la Carta de las Naciones como el deseo del concierto de naciones en fomentar las "relaciones de amistad basadas en el respeto al principio de igualdad de derechos y al de la libre determinación de los pueblos, y tomar otras medidas adecuadas para fortalecer la paz universal."

Es la Resolución 1514 emitida por la Asamblea General de las Naciones Unidas, documento conocido históricamente como la Declaración sobre la Concesión de la Independencia a los Países y Pueblos Coloniales (Gonzalez Campos, Sanchez Rodríguez, \& Saenz de Santa María, 2008, pág. 891) del 14 de diciembre de 1960, define este principio como la potestad que todos los pueblos tienen para el derecho de libre determinación; en virtud de esto, determinan libremente su condición política y persiguen libremente su desarrollo económico, social y cultural.

La misma resolución proclama que todos los pueblos tienen el derecho de determinar libremente, sin injerencia externa, su condición política y de proseguir su desarrollo económico, social y cultural.

No se autoriza ninguna acción contra la integridad territorial de Estados soberanos e independientes que se conduzcan de conformidad con el principio de la igualdad de los derechos y de la libre determinación de los pueblos antes descritos y estén, por tanto, dotados de un gobierno que represente a la totalidad del pueblo, perteneciente al territorio, sin distinción por motivos de raza, credo o color.

Partiendo de estas premisas mínimas para poder comprender de forma general el principio de autodeterminación de los pueblos, se menciona a partir de este punto, el nuevo enfoque de la problemática internacional al hablar sobre la unidad nacional en cuanto a la organización política de un Estado, específicamente al referirse al caso de Venezuela, la Resolución 1514 en su apartado sexto determina que: "todo intento encaminado a quebrantar total o parcialmente la unidad nacional y la integridad territorial de un país es incompatible con los propósitos y principios de la Carta de las Naciones Unidas.”

A través de la historia del Derecho Internacional han existido referencias que han ido encaminadas a defender la integridad territorial, estando totalmente en contra de la Secesión territorial, pero la Comunidad Internacional con la crisis actual en Venezuela, ha creado otra figura internacional a considerar, y es la integridad política del gobierno de un Estado desde la perspectiva que engloba la unidad nacional, al existir dualidad en la representación máxima del Estado venezolano con Nicolás Maduro por una parte y Juan Guaidó por otro lado.

La resolución 1514 si bien es cierto referencia la unidad nacional como parte de la autodeterminación del pueblo venezolana, se interpreta que la misma resolución enarbola la unidad política de un solo gobierno en un Estado. En consideración de las relaciones internacionales, desde la década de los sesentas, viendo la evolución de la realidad mundial, la aparición de problemas nuevos en el ámbito internacional o resurrección de ya existentes como ser los problemas demográficos, económicos culturales, ecológicos y energéticos, estamos ante la innovación de la problemática internacional en el plano político, desarrollando concepciones de las relaciones internacionales monopolizadas por conflictos armados, ahora son sustituidos por problemáticas propias de la evolución de la normativa internacional.

Resulta importante resaltar la tipología de autodeterminación que existen a partir del dictamen que el Tribunal Supremo de Canadá emite el 20 de agosto de 1998 en el asunto de la secesión de Quebec (Gonzalez Campos, Sanchez Rodríguez, \& Saenz de Santa María, 2008, pág. 903) afirmó:

"Las fuentes del derecho internacional reconocidas establecen que el derecho de un pueblo a disponer 
de sí mismo se realiza normalmente por vía de la autodeterminación interna, es decir, la persecución por parte de ese pueblo de su desarrollo político, económico, social y cultural en el marco de un Estado existente. El derecho a la autodeterminación externa..., no nace sin no en casos extremos, y en circunstancias por otra parte cuidadosamente definidas.

Es correcto determinar que la autodeterminación interna no representa una problemática jurídica para cada Estado en cuanto se respete la legalidad y los procesos internos enmarcados en el ámbito de un Estado de Derecho, siendo la autodeterminación externa, las más compleja en regular ya que puede desarrollarse desde la perspectiva del mismo país que implementa su autodeterminación interna, hacia una política exterior que puede tener una repercusión tanto positiva como negativa en sus relaciones internacionales, pero también surge el fenómeno de la autodeterminación externa como parte del mecanismo evolutivo de este principio en el ámbito internacional, de que la autodeterminación provenga de influencias impuestas por un tercer país en relación con otro, interpretándose como una injerencia relativa en asuntos internos de un Estado, tomando en consideración si se respeta o no la "voluntad soberana" de manifestaciones públicas de un sector de la población, en otorgar un reconocimiento internacional a un gobernante que no ha sido electo por el pueblo y deslegitimando un gobernante proveniente de elecciones internas, estén estas cuestionadas o no.

A partir de la premisa del "clamor popular" de una parte de la población inconforme con la situación interna de su propio país, enfrentando limitaciones en sus derechos humanos, las potencias mundiales de hoy en día, se escudan ante esta responsabilidad mesiánica de escuchar las voces que los gobernantes de estos no están dispuestos a tomar en consideración, otorgando legitimidad a su injerencia en los asuntos internos de determinada nación, dando nacimiento a otro principio internacional que es el punto de inflexión a la paz y seguridad internacional.

\section{Principio de Responsabilidad de Proteger}

La práctica internacional nos ha demostrado que ante estas situaciones lo más práctico y normal a ejecutar por la comunidad internacional en cuanto existen violaciones de derechos humanos en un Estado, pueden suscitarse las siguientes acciones previo a una intervención con uso de la fuerza para cumplir el principio de la responsabilidad de proteger:

Sanciones internacionales de índole diplomáticas económicas y embargos comerciales, (siempre que no implique el uso de la fuerza), contra el Estado, que de forma grava haya violado los derechos humanos esenciales (Gonzalez Campos, Sanchez Rodríguez, \& Saenz de Santa María, 2008, págs. 872-873) como ha sucedido en Venezuela con las sanciones económicas a la Petróleos de Venezuela (PDVSA), y congelamientos de cuentas offshore de Venezuela en Estados Unidos.

Sin embargo, hay 2 antecedentes históricos en la década de los noventa del principio de responsabilidad de proteger con resultados negativos y nefastos tanto para las Naciones Unidas, y la potencia mundial forjadora de este principio, Estados Unidos como lo es Ruanda y Somalia. En ambos países africanos, se consumó de forma devastadora el delito internacional de genocidio, pero la ONU y Estados Unidos en ambos escenarios tuvieron 2 actitudes diferentes y desconcertantes. El primero, fue pasado por alto y con acciones pasivas de parte de la ONU y Estados Unidos, quienes se inclinaron por acceder a proteger a la población mediante una misión de paz. En el caso de Somalia, (Hobsbawm, 2008, pág. 555), si se realizó una intervención militar por parte de Estados Unidos amparado en la obligatoriedad de proteger a la población víctima de genocidio, y retornar el país a la senda democrática, situación que fue un total fracaso por no existir un propósito claro por parte de Estados Unidos con la ONU, al no haber indicios de avances significativos en proteger a la población por las represalias armadas de las fuerzas belicosas que tenían inmersa a Somalia en una crisis política y humanitaria sin precedentes. 
Estos 2 escenarios dieron origen a que cualquier Estado podría plantear ante los órganos competentes la amenaza para la paz que se deriva de un incumplimiento grave de los derechos humanos, para que se adopten las medidas previstas en la Carta de las Naciones Unidas. Pero, además, importa señalar que un Estado puede recurrir a contramedidas o acciones unilaterales contra el infractor como se detalló anteriormente, pues si bien: "el empleo de la fuerza no puede ser un método apropiado para controlar y asegurar el respeto de tales derechos".

A partir de las acciones suscitadas en la década de los noventas e inicios del siglo XXI, de genocidios en África, Los Balcanes, Guerra del Golfo, Guerra de Irak, y Afganistán, fue en la Cumbre Mundial de Nueva York en el año 2005, que apertura una nueva dimensión al consagrar el concepto de responsabilidad de proteger, mediante el cual los Estados miembros de las Naciones Unidas se muestran dispuestos a proteger a las poblaciones del genocidio, crímenes de guerra, depuración étnica y crímenes de lesa humanidad. Estas acciones están encaminadas a aplicarse según la disposición a tomar medidas colectivas de manera oportuna y decisiva para tal fin, por medio del Consejo de Seguridad de conformidad con la Carta de las Naciones Unidas, incluido su Capítulo VII.

\section{Hipótesis de la Dualidad Política o Doble Gobierno en Venezuela}

El génesis de la crisis venezolana se da a partir del año 2013 con las elecciones celebradas en ese año, desconociendo la oposición el resultado que da como vencedor al sucesor de Hugo Chávez, Nicolás Maduro en detrimento del candidato de la oposición, Henrique Capriles. Desde este momento en particular podemos delimitar el principio de una crisis agudizada en este año 2019.

Al verse obligada la oposición a una aceptación tácita de los resultados de esa elección del año 2013, vemos que, en el año 2017, instaura el presidente Maduro la Asamblea Nacional Constituyente para la reestructuración del Estado venezolano y la redacción de una nueva constitución. Se trae a colación este hecho, ya que esto es una voluntad propiamente de una efectiva autodeterminación interna de un país en establecer una reingeniería estatal, sin embargo, este hecho da un paso más hacia la crisis interna de Venezuela, sentando un precedente en un una situación internacional de tener una incidencia antagónica en el seno de Naciones Unidas, teniendo como protagonistas directos a las 3 potencias, que son miembros permanentes del Consejo de Seguridad de la ONU, por un lado, Rusia y China que apoyan directamente al Gobierno de Nicolás Maduro.

Es así que Venezuela, define su postura en la libertad del pueblo venezolano como parte de su autodeterminación al esclarecer su futuro político, acatando la comunidad internacional, la voluntad soberana del pueblo venezolano, y por otro lado, Estados Unidos quien niega rotundamente reconocer al Presidente Maduro como el titular del Ejecutivo por las flagrantes violaciones a los derechos humanos del pueblo de Venezuela al escasear deliberadamente como señal de opresión los servicios básicos (agua, luz, gas, alimentos) para la subsistencia de los pueblos, brindando su apoyo incondicional al auto proclamado Presidente Interino de Venezuela Juan Guiadó, sugiriendo de forma indirecta que la opción armada no esta descartada en Venezuela como responsabilidad de proteger al pueblo.

Lo que sobre sale de esta acción propia del pueblo venezolano representados por sus líderes políticos emanados de elecciones que múltiples países del concierto internacional en aplicación de su libre autodeterminación externa, desconocen la instauración de esta asamblea nacional constituyente, afirmando que es un rompimiento del orden democrático en Venezuela, pero más sorprende la rapidez en que la comunidad internacional ha reaccionado para reconocer o deslegitimar un gobierno electo "democráticamente", existiendo siempre los fantasmas de las irregularidades electorales como sucedió en Honduras.

En este punto, es importante acotar que, al existir un patrón trazado en toda América Latina en cuanto a la reelección presidencial, el informe preliminar de la 
Misión de Observación Electoral (MOE) de la OEA en Honduras, destacó que en cuanto a la regulación sobre reelección presidencial "constituye una mala práctica que se ha presentado en otros países de la región", pudiéndose interpretar como una "epidemia ideológica” en política interna de muchos Estados de la región en aplicar una reelección presidencial mediante sentencias de cortes constitucionales con fundamentación tergiversada de jurisprudencia de convenios internacionales en materia de Derechos Humanos, alegando estarse afectando el derecho a la igualdad como ciudadano, a ser electo y a la libre representación, y la libre participación en condiciones de igualdad en justas presidenciales.

"La misión entiende que los derechos políticos no son absolutos. En este contexto, señala que sí admiten restricciones siempre que se encuentren previamente en una ley y no sean abusivas o arbitrarias y que cumplan con los requisitos de idoneidad, necesidad y proporcionalidad." (OEA, 2017).

Los conflictos que actualmente enfrente Venezuela y que otros países adolecen en mayor o menor medida por aplicarse la reelección presidencial, si bien es cierto se contemplan en las normativas internas de los Estados, se aplica de forma inversa como lo establece la MOE de forma abusiva para perpetrarse de en el poder, conocidas como "dictaduras democráticas".

Es congruente afirmar que la temática reeleccionista en Latinoamérica, deriva o propicia atentar contra el principio internacional de libre determinación de los pueblos, en virtud de no ser el pueblo quien decide el futuro de sus gobernantes, si no los gobernantes el futuro de su población, derivando este patrón en que en algún determinado momento, alguna potencial mundial pueda propiciar una aplicación del principio de responsabilidad de proteger, por la incapacidad de los gobernantes en mantener la unidad política, y la proliferación de acciones que puedan constituir hechos internacionalmente ilícitos que allanen la posibilidad de la intervención del uso de la fuerza en base al compromiso internacional por proteger a la población.
En la secuencia de los hechos, en el año 2018, con las elecciones presidenciales en Venezuela, teniendo como actores principales al Presidente en funciones, Nicolás Maduro, optando a su reelección por segundo período consecutivo, relacionando que los períodos presidenciales en Venezuela son de 7 años y no de 5 como en Honduras, obteniendo una victoria abrumadora contra sus opositores; cuestionando la comunidad internacional los resultados, que no tuvieron una observación electoral por parte de Naciones Unidas ni por parte de la OEA, organismo internacional regional que en su sesión cuadragésima octava sesión de la Asamblea General celebrada el 4 y 5 de junio del 2018 en Washington D.C. (Organización de Estados Americanos, 2018), ya comienza a trazar una definición de situación política en Venezuela, calificándola de una crisis económica, política, social y humanitaria, declarando que el proceso electoral carece de legitimidad.

El 23 de enero del 2019 quedará marcada como la fecha del nacimiento del novedoso, y en ciertos aspectos del derecho internacional, la tenebrosa figura antagónica del principio de unidad nacional enarbolado en la Resolución 1514 de la ONU, con la hipótesis o principio de dualidad política en el marco del derecho internacional, al autoproclamarse Juan Gerardo Antonio Guaidó Márquez, líder opositor del chavismo, quien fungía como Presidente de la Asamblea Nacional de Venezuela (Poder Legislativo), como Presidente Interino de Venezuela como lo establecen los artículos 233, 333 y 350 de la Constitución venezolana (Partido Solicalista Unido de Venezuela, s.f.) que literalmente manifiestan:

"Serán faltas absolutas del Presidente o Presidenta de la República: la muerte su renuncia, la destitución decretada por sentencia del Tribunal Supremo de Justicia, la incapacidad física o mental permanente certificada por una junta médica designada por el Tribunal Supremo de Justicia y con aprobación de la Asamblea Nacional, el abandono del cargo, declarado éste por la Asamblea Nacional, así como la revocatoria popular de su mandato." 
"Esta Constitución no perderá su vigencia si dejare de observarse por acto de fuerza o porque fuere derogada por cualquier otro medio distinto el previsto en ella. En tal eventualidad, todo ciudadano investido o ciudadana investida o no de autoridad, tendrá el deber de colaborar en el restablecimiento de su efectiva vigencia.

"El pueblo de Venezuela, fiel a su tradición republicana, a su lucha por la independencia, la paz y la libertad, desconocerá cualquier régimen, legislación o autoridad que contrarie los principios y garantías democráticas o menoscabe los derechos humanos."

A partir de estos acontecimientos descritos y de otros sucesos que han recrudecido la crisis venezolana, las naciones del concierto internacional, reconocen de manera casi inmediata a Juan Guaidó como presidente interino de Venezuela, siendo a día de hoy un total de 54 países (Panam Post, s.f.) los que reconocen su estatus: Albania, Alemania, Andorra, Argentina, Austria, Australia, Bahamas, Bélgica, Brasil, Bulgaria, Canadá, Chile, Chipre, Colombia, Costa Rica, Croacia, Dinamarca, Ecuador, España, Estados Unidos, Estonia, Finlandia, Francia, Georgia, Guatemala, Haití, Honduras, Hungría, Irlanda, Islandia, Israel, Kosovo, Letonia, Lituania, Luxemburgo, Macedonia, Malta, Marruecos, Montenegro, Países Bajos, Panamá, Paraguay, Perú, Polonia, Portugal, Reino Unido, República Checa, República Dominicana, Rumania, Suecia, Suiza, Taiwán, y Ucrania. Por parte de Nicolás Maduro, ostenta el reconocimiento internacional de Rusia, China, Irán, Cuba, Bolivia, Nicaragua, Turquía y Namibia. En posición neutral, México, Uruguay, Italia, Noruega y El Vaticano.

De este listado de Estados con su aceptación de un presidente y otro, queda consumado el antecedente internacional contra la unidad nacional en el ámbito político, que los Estados de la comunidad internacional están en su responsabilidad de respetar, pero que tiene su antagonismo con la Hipótesis de la Dualidad Política con estas determinaciones en sus políticas exteriores de estas naciones con la crisis venezolana.
Al ostentar el beneplácito de gran parte de la comunidad internacional en detrimento de Nicolás Maduro, el 30 de abril 2019 (Cable News Network en Español, s.f.), Juan Guaidó convoca a la población a ejecutar la fase final de la Operación Libertad, para poder sacar a Nicolás Maduro del poder. Dicho en otras palabras, un golpe de Estado de un presidente a otro, con supuesto apoyo de Estados Unidos que podemos catalogarlo como el autor material e intelectual del principio o hipótesis de la dualidad política en Venezuela, por haber sido el primero en reconocer a Guaidó como presidente interino.

Al haberse frustrado el éxito de la Operación Libertad, se da el conflictivo diplomático entre Estados Unidos, Rusia y China, viendo la oportunidad de estos últimos que el plan fallido de Estados Unidos para poder tener un control más directo de Venezuela por sus intereses económicos en cuanto al petróleo se refiere, por ser de reconocida costumbre, que Estados Unidos considera su "patio trasero" a América Latina; estos últimos han adoptado una postura menos beligerante en cuanto a sus políticas exteriores con relación a Venezuela por sus intereses comerciales, ya que Maduro ha podido mantener su gobierno por las ayudas y relaciones petrolíferas que mantiene con China y Rusia, y así poder disminuir la injerencia "imperialista americana" en Venezuela como principal objetivo de China.

Por parte de Rusia, su injerencia en Venezuela se puede interpretar como una "vendetta" por parte del Kremlin por las injerencias que ha ejercido Estados Unidos en Ucrania y Georgia, que ha conllevado a múltiples problemas a Rusia en los ámbitos territoriales, políticos, y diplomáticos con estos países. En otras palabras, es una lucha de "titanes" para poder establecer quien manda en Latinoamérica y en el mundo, por una parte, si Maduro permanece en el poder, se interpretaría como el nacimiento de una nueva potencia hegemónica no solo en Venezuela, sino a nivel regional, que puede mantener gobiernos con amenazas disuasivas hacia Estados Unidos para no utilizar el uso de la fuerza como ya sucedió en Siria. En caso de que Maduro abandone el poder, se confirmaría la tesis de patio trasero de Estados Unidos 
en Venezuela y Latinoamérica, y derrocar gobiernos con sus políticas coercitivas contra el sistema de paz internacional ya sea mediante golpes de estado, o deslegitimación de gobiernos bajo la premisa de imposición de gobernantes interinos bajo el principio o hipótesis de dualidad política.

La OEA en fecha 29 de junio del 2019 emite adopta una nueva resolución en cuanto a la degradada crisis en la república bolivariana, la cual determinó que es imperativo restaurar el orden democrático en Venezuela con la convocatoria de elecciones transparentes y buscar soluciones para la problemática migratoria del éxodo de venezolanos por la agudización de la vulneración de derechos civiles, sociales y culturales como ser escasez de agua, luz, gas, petróleo e insumos alimenticios. Estos últimos son considerados como derechos de segunda generación con respecto a los derechos civiles y políticos en base a 2 criterios fundamentales. Los derechos civiles y políticos se consideran derechos negativos que imponen a los Estados obligaciones de no hacer, mientras que los derechos económicos, sociales y culturales son obligaciones positivas o de hacer. Referido en otras palabras, los primeros se aplican de forma inmediata por la facilidad del Estado en cumplirlos, mientras que los segundos, requieren un esfuerzo financiero por parte del Estado, siendo aplicables en forma progresiva (Romani, y otros, 2007, pág. 163), por ende, la escasez de insumos como parte de los derechos humanos, se evalúa dependiendo de la disposición financiera del Estado para cumplirlos, en el caso de Venezuela, existe la escasez por las presiones y sanciones económicas a Maduro por Estados Unidos, no porque el Estado Venezolano no pueda abastecerlas.

Las posturas que actualmente profesan las potencias mundiales como Rusia y China, es que al pueblo venezolano debe respetársele sus decisiones en función de sus asuntos internos como parte de su libre determinación como lo establece la Carta de las Naciones Unidas en su artículo 2 párrafo 7. "Provoca mucha discusión este principio ya que se le quiere dar una gran seguridad a los países, sobre todo a las grandes potencias, de que las Naciones Unidas no penetrarían en el llamado dominio reservado, para garantizar su propia soberanía, pero si quieren otorgar potestad a la ONU para recurrir a potencias mundiales cuando el caso lo amerite para violentar la soberanía de los países no consideradas potencias mundiales.” (Sepulveda, 2000, pág. 299)

Estados Unidos por su parte siempre ha sido una nación como se ha detallado a lo largo de este artículo, de poseer un carácter impositivo al momento del uso de la fuerza, y como principal precursor del Principio de Responsabilidad de Proteger en consonancia con la hipótesis de la dualidad política en detrimento de la unidad nacional de un país. En cuanto a la postura norteamericana se refiere ante la problemática de la República Bolivariana, esta ha sido exteriorizada por parte de su Secretario de Estado Mike Pompeo y el asesor de Seguridad Nacional John Bolton (British Broadcasting Company, s.f.), de nunca descartarse la opción militar en Venezuela, como mecanismo para la responsabilidad internacional que tiene Estados Unidos, impulsado por sus propios intereses económicos y geo-políticos (Petróleo, Recursos Naturales Renovables y No Renovables, posicionamiento militar estratégico) de proteger al pueblo venezolano de la opresión.

A día de hoy la crisis o situación venezolana como se le quiera llamar, se encuentra en un punto muerto, entre el respeto y apoyo al principio de libre autodeterminación del pueblo venezolano a continuar con el régimen chavista con Nicolás Maduro, o apoyar a un presidente interino amparado en la obligación legal y moral de encaminar a Venezuela a ser nuevamente un Estado de Derecho, y que sean estos los que resuelvan sus propios conflictos respetando el principio de no injerencia en los asuntos internos de un Estado, o por otra parte, esperar el momento idóneo para poder justificar mediante el principio de responsabilidad de proteger por acciones lesivas a los derechos humanos de la población, una incursión militar que podría desencadenar un conflicto armado entre 2 potencias históricas que desde la Guerra Fría, aún no han entibiado sus deseos hegemónicos de controlar y dominar el globo terráqueo, y China al acecho de convertirse en una potencia mundial sin recurrir al uso de la fuerza. 


\section{CONCLUSIONES}

- La evolución de principios o acciones que atentan contra el derecho internacional se deriva por el interés político, económico o social de una potencia mundial para la explotación o aprovechamientos de estos en relación a los avances que pueda tener un país de menor categoría.

- El nacimiento del Principio o Hipótesis de la Dualidad Política en detrimento de la unidad nacional de un país, advierte un cierto grado de peligrosidad de convertirse en costumbre internacional de las grandes potencias, atentando directamente al principio de injerencia en asuntos internos de las grandes potencias en países subdesarrollados o en vías de desarrollo.

- El derecho a la autodeterminación de los pueblos en relación con el principio de responsabilidad de proteger continúa siendo un principio de primera categoría en el derecho internacional, y se relaciona el segundo como el preludio a conflictos armados que resquebrajen la paz y seguridad internacional, atentando contra el orden internacional del respeto "erga omnes" de los derechos humanos en el caso particular de Venezuela o de cualquier otro Estado del concierto internacional en su autodeterminación tanto interna como externa.

- La crisis interna de Venezuela ha demostrado que el derecho internacional, las organizaciones y sus miembros, aún no poseen la madurez suficiente para adoptar posturas jurídicas sólidas, más allá de simples declaraciones o reconocimientos sin sentido, sin brindar soluciones factibles a problemáticas internas de los Estados con posibles repercusiones internacionales.

\section{BIBLIOGRAFÍA}

- British Broadcasting Company. (s.f.). Obtenido de BBC: https://www.bbc.com/mundo/noticiasamerica-latina-48128434
- Cable News Network en Español. (s.f.). Obtenido de CNN en Español: https://cnnespanol.cnn. com/2019/07/22/se-cumplen-6-meses-de-laproclamacion-de-guaido-cronologia/

- del Arenal Moyua, C. (2007). Introducción a las Relaciones Internacionales. Madrid: Tecnos.

- Gonzalez Campos, J. D., Sanchez Rodríguez, L. I., \& Saenz de Santa María, P. A. (2008). Curso de Derecho Internacional Público. Pamplona: Civitas.

- Hobsbawm, E. (2008). Historia del Siglo XX. Barcelona: Critica.

- OEA. (4 de diciembre de 2017). El Heraldo. Obtenido de https://www.elheraldo.hn/ csp/mediapool/sites/dt.common.streams. StreamServer.STREAMOID $=x b A S v F E m k c$ vSGZvYi072cpM5tm0Zxrvol3sywaAHBAm Kp1GRebW2VnE8dZjZNua\$8vdSm1Hx2Y WUgDC4QYKEka4nGJOwGe0EnkFAoFi M1vw2wnj7OUsk04 0foOmFCqtrvCWnkLNFtL0tdmsB2s5BgvHsxoNcXRVPW

- Organización de Estados Americanos. (4 y 5 de Junio de 2018). Obtenido de OEA: https://www. oas.org/es/centro noticias/comunicado prensa. asp? $\mathrm{s} \operatorname{Codigo}=\mathrm{D}-032 / 18$

- Panam Post. (s.f.). Obtenido de https:// es.panampost.com/miguel-camacho/2019/02/12/ paises-reconocen-guaido/

- Partido Solicalista Unido de Venezuela. (s.f.). Obtenido de PSUV: http://www.psuv.org.ve/ constitucion/

- Romani, C. F., Soroeta Liceras, J., Bollo Arocena, M. D., Quel López, F. J., Ruiloba Alvariño, J., Pastor Palomar, A., . . Cano Linares, Á. (2007). Derecho Internacional de los Derechos Humanos. Madrid: Dilex.

- Sepulveda, C. (2000). Derecho Internacional. Mexico D.F.: Porrua. 